\title{
La radio aragonesa y el fracaso del Plan de Adjudicación de Frecuencias de 1998
}

\author{
Dr. Fernando Sabés Turmo \\ Profesor Asociado de la Facultad de Comunicación de la \\ Universidad Autónoma de Barcelona
}

\section{RESUMEN}

El Gobierno de Aragón concedió en 1998 un total de 32 frecuencias de radio destinadas a emisoras comerciales privadas. Entre las condiciones que principalmente se valoraron destacan dos: el intentar fomentar la pluralidad de las emisiones y el de favorecer a las empresas que apostaran por la programación propia.

Sin embargo, después de algo más de cuatro años se ha comprobado como las 32 frecuencias han ido a parar a grandes grupos mediáticos de forma directa o mediante acuerdos de colaboración, mientras que no se ha creado ningún operador que apueste claramente por una parrilla propia. Incluso hay algunas redes que todavía no han comenzado a emitir y otras que lo hacen únicamente como postes repetidores.

En este sentido, la reducida importancia de las emisoras municipales, de las radios sin licencia, de las desconexiones de Radio Nacional de España y, sobre todo, la falta de una radio pública regional, conlleva a la escasez de contenidos de proximidad de este medio en Aragón.

Se ha comprobado como el plan de adjudicación de frecuencias de 1998 ha fracasado en lo que era su principal objetivo: dinamizar la oferta radiofónica en Aragón. Solamente ha permitido consolidar a las grandes cadenas de radio estatales y a los nuevos proyectos que se han expandido en toda España en los últimos tiempos como son Radio Intereconomía y Flaix FM.

Visto este panorama, parece cada vez más necesario una apuesta por la Radio Pública Autonómica, que podría ver la luz en la próxima legislatura, según los movimientos políticos que se han producido en los últimos tiempos.

\section{ABSTRACT}

The Government of Aragón conceded 32 frequencies of radio in 1998 destined to commercial private radios. Between the conditions that mainly were valued highlights two: the attempt to foment the plurality of the emissions and the of favoring to the companies that they bet for the own code.

However, after something more than four years it has been checked like the 32 frequencies have been controlated by the big groups of media of direct form or by means of agreements of colaboration, while no operator has not been created that it bet clearly for an own grill. Including there are some nets that they have not begun to emit and anothers nets that make it only like posts repeaters.

In this sense, the reduced importance of the municipal radio, of the without license radios, of the disconnections of National Radio from Spain and, above all, the lacking of a public regional radio, imply to the scarcity of contents in vicinity of this medium in Aragón. 
It has been checked like the plan of award of frequencies of 1998 it has failed in his principal objective: invigorate the offer of the radio in Aragón. Only it has allowed to consolidate to the big chains of state radio and to the new projects that they are expanding in all Spain in the last times. They are Intereconomía Radio and Flaix FM.

Sees this panorama, it seems more and more necessary a bet for the Public Autonómica Radio, that it could appear in the next legislature, according to the political movements that have been produced in the last times.

Palabras claves: Radio/Aragón/Concesión y grandes grupos mediáticos.

Key words: Radio/Aragón/Concession and big groups of media.

\section{La radio aragonesa y el fracaso del Plan de Adjudicación de Frecuencias de 1998}

7 a adjudicación de frecuencias de radio llevada a cabo por el Gobierno de Aragón el 31 de junio de 1998 ha supuesto un rotundo fracaso en función de los objetivos que se había marcado el Ejecutivo regional con el desarrollo de este plan, sobre todo, en relación a la diversificación de la oferta radiofónica y a la potenciación de emisiones de proximidad, dos aspectos que después de algo más de cuatro años se ha comprobado como no se han cumplido.

Las 32 emisoras otorgadas han servido para consolidar la oferta de los grandes grupos radiofónicos (SER, COPE y Onda Cero), así como para impulsar nuevos proyectos a nivel estatal (Radio Intereconomía y Flaix FM), mientras que, por el contrario, no se contabiliza en la actualidad ni una sola iniciativa que, surgida tras estas concesiones, tenga como objetivo crear una radio aragonesa y sin relación con otras foráneas.

No obstante, en la Orden de 7 de noviembre de 1997, emitida por el Departamento de Ordenación Territorial, Obras Públicas y Transportes del Gobierno de Aragón, por la que se convocaba el concurso público para la asignación de concesiones del Servicio Público de Radiodifusión Sonora en Ondas Métricas con Modulación de Frecuencia, con destino a su gestión indirecta privada de carácter comercial se señalaba, en el anexo, que uno de los principales objetivos era el de potenciar el tejido comunicativo aragonés, apostando por la variedad de operadores y también por evitar que las nuevas frecuencias que se concederían al año siguiente se convirtieran en meros postes que reemitieran la señal de las programaciones de las grandes radios estatales. En el pliego de condiciones se recogía que estos puntos del dial se adjudicarían en función de siete criterios:

1.- La pluralidad de la oferta de radiodifusión sonora en ondas métricas con modulación de frecuencia en el ámbito de la concesión y en la totalidad de la Comunidad Autónoma de Aragón. 
2.- La viabilidad económica y técnica del proyecto, en relación con las inversiones previstas para la instalación y explotación de la emisora, utilizando preferentemente tecnología y equipos con valor añadido aragonés, y con el personal que prestará servicio con carácter fijo y dependencia directa del solicitante, y su continuidad durante el plazo de la concesión.

3.- El horario de emisión, tipo de programación, el porcentaje anual de programas, del total de la programación que se pretenda emitir, de producción propia y ajena, con especial referencia a los de tipo informativo, cultural o educativo, y de ellos los de interés peculiar en su zona de cobertura en el plazo de vigencia de la concesión, y el compromiso de mantener esa situación durante un plazo determinado.

4.- La cesión al Gobierno de Aragón para su uso o gestión, con carácter gratuito, de una parte del mástil soporte del array de antenas que vayan a ser usadas para transmisión, no coincidente con dicho array y en la zona mitad superior del soporte. Así como la cesión de una parte de los terrenos donde vayan a ubicarse los soportes antes referidos y del derecho de conexión al suministro eléctrico que exista en dichos puntos, en ambos casos con carácter gratuito.

5.- La solicitud de un número de frecuencias que garantice la utilización efectiva de las ubicadas en las cabeceras de comarca y menor potencia (P.R.A.W).

6.- El compromiso de no transferir ni ceder la concesión durante un período determinado, en exceso sobre el señalado en la Ley de Contratos de las Administraciones Públicas para este tipo de contratos, ni de variar la explotación de la concesión o la prestación del servicio en la forma propuesta en la solicitud de adjudicación.

7.- Otros criterios que, a la vista de la documentación presentada, puedan ser considerados especialmente relevantes.

La asignación de estos puntos del dial fue complicada. El Gobierno regional, formado en aquel momento por una coalición entre el Partido Popular (PP) y el Partido Aragonés (PAR), comenzó a estudiar las 271 propuestas formuladas en febrero de 1998. Sin embargo, nadie pujó por las cinco frecuencias en las localidades de Boltaña (Huesca), Alcalá de la Selva (Teruel), Moreal del Campo (Teruel), Montalbán (Teruel) y Belchite (Zaragoza). En ese momento se volvió a abrir un plazo para intentar que alguna sociedad se interesara por estas localidades, aspecto que finalmente se consiguió.

La Diputación General de Aragón (DGA) distribuyó las 32 frecuencias concedidas de la siguiente forma: doce en Huesca, nueve en Teruel y once en Zaragoza. 
Tabla 1. Frecuencias concedidas en 1998 en Aragón

\begin{tabular}{|c|c|c|c|}
\hline Provincia & Localidad & Frecuencia & Adjudicataria \\
\hline Huesca & Barbastro & 106.9 & Radio Popular \\
\hline Huesca & Benabarre & 101.1 & José Luis Brualla \\
\hline Huesca & Benasque & 103.1 & Antena del Pirineo \\
\hline Huesca & Canfranc & 90.3 & Ambiente Musical \\
\hline Huesca & Castejón de Sos & 101.7 & Raúl Joaquín Urrea \\
\hline Huesca & Fraga & 93.1 & Radiofonía Aragonesa \\
\hline Huesca & Huesca & 106.0 & Publicidad 3 \\
\hline Huesca & Huesca & 98.2 & Radio Popular \\
\hline Huesca & Jaca & 106.6 & Radio Popular \\
\hline Huesca & Monzón & 93.8 & Ixalencas \\
\hline Huesca & Sabiñánigo & 99.5 & Uniprex \\
\hline Huesca & Boltaña & 100.4 & Cadena Voz de Radiodifusión SA \\
\hline Teruel & Alcañiz & 102.9 & Promotora Cultural del Bajo Aragón \\
\hline Teruel & Andorra & 105.3 & Radiofonía Aragonesa \\
\hline Teruel & Calamocha & 87.6 & Uniprex \\
\hline Teruel & Mora de Rubielos & 102.6 & Sara-Buy 6000 \\
\hline Teruel & Teruel & 106.7 & Radio Popular \\
\hline Teruel & Teruel & 99.6 & Prensa Española de Radio por Ondas \\
\hline Teruel & Alcalá de la Selva & 92.3 & Cadena Voz de Radiodifusión \\
\hline Teruel & Monreal del Campo & 92.0 & Cadena Voz de Radiodifusión \\
\hline Teruel & Montalbán & 104.1 & Cadena Voz de Radiodifusión \\
\hline Zaragoza & Calatayud & 105.4 & Comunicación Calatayud \\
\hline Zaragoza & Cariñena & 95.7 & Canal Mundo Radio Aragón \\
\hline Zaragoza & Ejea de los Caballeros & 98.1 & Central Aragonesa de Producción y Medios \\
\hline Zaragoza & Mequinenza & 95.5 & Luis del Olmo \\
\hline Zaragoza & Tarazona & 99.1 & Cadena Voz de Radiodifusión \\
\hline Zaragoza & Tauste & 101.3 & Heraldo de Aragón \\
\hline Zaragoza & Utebo & 87.6 & Radiofonía Aragonesa \\
\hline Zaragoza & Zaragoza & 89.7 & Central Aragonesa de Producción y Medios \\
\hline Zaragoza & Zaragoza & 100.5 & Publicidad 3 \\
\hline Zaragoza & Zuera & 103.2 & Radio Zaragoza \\
\hline Zaragoza & Belchite & 87.9 & Cadena Voz de Radiodifusión \\
\hline
\end{tabular}

*Elaboración propia. Fuente: Gobierno de Aragón 
De estas 32 adjudicaciones llevadas a cabo en el verano de 1998 solamente nueve fueron destinadas a sociedades o personas que a priori no estaban vinculadas a grandes grupos mediáticos. En concreto, las siguientes: Benabarre (José Luis Brualla), Benasque (Antena del Pirineo), Castejón de Sos (Raúl Joaquín Urrea), Monzón (Ixalencas), Alcañiz (Promotora Cultural del Bajo Aragón), Mora de Rubielos (Sara-Buy 6000), Calatayud (Comunicación Calatayud), Ejea de los Caballeros y Zaragoza (Central Aragonesa de Producción y Medios).

Asimismo, de estas nueve concesiones, en la actualidad todavía hay una que no ha comenzado a funcionar después de más de cuatro años de haberse resuelto el concurso: Mora de Rubielos (Sara-Buy 6000).

Las ocho restantes iniciaron su andadura de forma muy diversa. Cinco llegaron rápidamente a acuerdos con la SER y la COPE.

José Luis Brualla comenzó a emitir en Benabarre, en el 101.1, programación de Cadena Dial; Antena del Pirineo, en el 103.1 en Benasque, de la COPE; Ixalencas, en el 93.8 en Monzón, de Cadena 100; Promotora Cultural del Bajo Aragón, en el 102.9 en Alcañiz, de Cadena 100; y Comunicación Calatayud, en el 105.4 en Calatayud, de la COPE.

Solamente dos adjudicatarios, Raúl Joaquín Urrea en Castejón de Sos (101.7) y Central Aragonesa de Producción y Medios en Zaragoza (89.7) iniciaron sus emisiones con una parrilla propia y desvinculada de alianzas con otros operadores estatales. Hay que indicar que el segundo punto en el dial concedido a Central Aragonesa de Producción y Medios en Ejea de los Caballeros (98.1) no se puso en funcionamiento hasta finales de 2001. Pero la existencia de estos dos proyectos fue fugaz ya que ambos centros acabaron en poco tiempo ligados a redes estatales.

Pirineos Música fue la denominación de la estación que se puso en marcha en el 101.7 de Castejón de Sos. Era una iniciativa que prácticamente sólo funcionó en periodo de pruebas y que acabó por desaparecer al llegar a un acuerdo su adjudicatario con Onda Cero y en marzo de 2002 pasó a transmitir la programación generalista de la emisora de Admira.

Por su parte, Central Aragonesa de Producción y Medios impulsó la creación de Antena Aragón Radio en Zaragoza (89.7), un proyecto que se centró en la incorporación masiva de música, aunque combinada con una mínima presencia de información y deporte. Sin embargo, en julio de 2001 pasó a emitir la programación de Radio Intereconomía, grupo que en el último año ha incrementado de forma notable su presencia en todo el Estado a través de diferentes alianzas y compra de frecuencias. En este sentido, mediante la Orden de 9 de junio de 2002 del Departamento de Obras Públicas, Urbanismo y Transportes del Gobierno de Aragón se autorizó la transferencia de la titularidad de las dos concesiones en Zaragoza y Ejea, adjudicadas en 1998 a Central Aragonesa de Producción y Medios, a la sociedad Intereconomía Aragón S.L. 
Con el adiós a estos dos proyectos se puede decir que también han desaparecido las dos únicas iniciativas que surgidas tras las asignaciones de 1998 buscaban la creación de un producto radiofónico de carácter aragonés y sin vinculación con los operadores estatales.

Las 23 frecuencias restantes se distribuyeron de la siguiente forma: cuatro a Radio Popular (Teruel, Barbastro, Huesca y Jaca); tres a Radiofonía Aragonesa, en un primer momento la de Zaragoza emitió Z Radio Onda Corazón, y después se incorporaron a Flaix FM (Fraga, Utebo y Andorra); diez vinculadas a Onda Cero a través de tres sociedades: Publicidad 3, Uniprex y Cadena Voz de Radiodifusión (Huesca, Sabiñánigo, Boltaña, Calamocha, Alcalá de la Selva, Monreal del Campo, Montalbán, Tarazona, Zaragoza y Belchite); una a Radio Zaragoza SER (Zuera); y otra a Ambiente Musical (Canfranc). Además, el 99.6 de Teruel, asignado a Prensa Española de Radio por Ondas, se convirtió en Cadena 100 Teruel; el 95.7 de Cariñena, propiedad de Canal Mundo Radio Aragón, pasó a ser un repetidor de Onda Cero; y el 101.3 de Tauste, adjudicado a Heraldo de Aragón, se vinculó a la SER. Asimismo, el 95.5 de Mequinenza, ha funcionado irregularmente en pruebas y después de emitir la programación musical de Hit Ràdio se ha convertido, aunque parece que de momento de forma provisional, en un repetidor de Onda Rambla Lleida-Cadena Onda Cero.

De las 32 adjudicaciones, todavía en la actualidad hay seis que no han iniciado sus emisiones: Canfranc (90.3), Boltaña (100.4), Mora de Rubielos (102.6), Monreal del Campo (92.0), Montalbán (104.1) y Belchite (87.9). Cuatro de ellas están asignadas a Cadena Voz, una a Ambiente Musical (Canfranc) y otra a SaraBuy 6000 (Mora de Rubielos).

Asimismo, no todos los operadores emiten desde el lugar donde se otorgó la frecuencia. Hay dos casos evidentes que incumplen lo establecido en el concurso. Onda Cero transmite en Teruel capital por el 92.3 de Alcalá de la Selva al haber tenido que ceder a Kiss FM el 101.6. Asimismo, Radiolé utiliza la frecuencia adjudicada a Radio Zaragoza en Zuera (103.2), pero en este caso, en el mismo punto en el dial, se emiten dos programaciones diferentes Radiolé Zaragoza y Radiolé Zuera, aunque esta última con una potencia limitada y destinada únicamente a cubrir esta segunda población. Es decir, la primera parrilla pisa a la segunda salvo en la localidad de Zuera.

Por otro lado, hay que señalar que el 93.1 de Fraga, asignado a Radiofonía Aragonesa y por el que se sintoniza actualmente Flaix FM, está destinado básicamente a dar cobertura mediante esta programación musical a la provincia de Lleida. En este sentido, el idioma utilizado es el catalán. Este fenómeno, que se produce por la proximidad de Fraga con Cataluña, se da también en el 95.5 de Mequinenza, que de momento es un repetidor de Onda Rambla Lleida-Cadena Onda Cero.

De esta forma, se puede señalar que el plan de concesión de frecuencias desarrollado por el Gobierno de Aragón en 1998 solamente ha servido para 
consolidar la posición de privilegio de las cadenas estatales en la Comunidad Autónoma, así como ha facilitado la aparición de algunos otros operadores que se están extendiendo también en el resto de España, pero, en ningún momento, ha permitido dinamizar la oferta radiofónica aragonesa que ha quedado relegada únicamente a las mínimas desconexiones que desarrollan estos centros a lo largo de la jornada y a la programación que ofrecen las radios municipales y las sin licencia, ya que el caso de Radio Nacional de España es muy similar al de las redes comerciales.

Prácticamente no se ha cumplido ninguno de los puntos realmente importantes recogidos en los criterios que se tenían que valorar para la otorgación de estos puntos del dial en 1998. A continuación repasaremos los siete elementos que se debían tomar en consideración:

1.- Pluralidad de la oferta: lo que se ha logrado con este plan ha sido consolidar las programaciones de las redes estatales, ya sea mediante la adjudicación directa a estos grupos o a través de los acuerdos posteriores, aunque alguno ya se había negociado anteriormente, con las personas y sociedades que finalmente recibieron la autorización para emitir. Bajo ningún concepto se puede decir que se ha logrado diversificar la oferta. Sí es cierto que han aparecido en el espectro radiofónico aragonés nuevas cadenas, como es Radio Intereconomía y Flaix FM, pero son emisoras que se han expandido en prácticamente todo el Estado y que realizan una programación de proximidad reducida.

\section{2.- Viabilidad técnica y económica del proyecto y personal contratado:} la inversión ha sido muy escasa tanto en instalaciones como en personal, sobre todo, porque estas nuevas frecuencias se han convertido en repetidores de señal y plantean mínimas desconexiones locales, que en buena parte de los casos no llegan a producirse.

3.- Programación propia e implicación en la zona de cobertura: como ya hemos señalado la mayor parte de estas concesiones se han convertido en postes repetidores. En algunos casos sí realizan desconexiones a lo largo de la jornada, mínimas en su mayoría, pero en otros solamente se dedican a difundir la señal, y la única programación local que incorporan es la publicidad. El hecho de que muchas de estas frecuencias se hayan dedicado a la radio musical ha impedido, en buena parte, crear una parrilla de proximidad que era lo que se reclamaba en este punto del pliego de condiciones.

Estos eran los tres aspectos principales que se debían valorar, ya que los otros cuatro eran criterios que cualquier propuesta podía asumir. Simplemente se requería el compromiso de la entidad aspirante: 
4.- Cesión al Gobierno de Aragón de una parte del mástil soporte del array de las antenas y de una parte de los terrenos.

5.- La solicitud de un número de frecuencias que garantice la utilización efectiva de las ubicadas en las cabeceras de comarca y menor potencia.

6.- El compromiso de no transferir ni ceder la concesión durante un periodo determinado.

7.- Otros elementos que, a la vista de la documentación presentada, puedan ser considerados especialmente relevantes.

Los tres primeros aspectos podían sumar un máximo de 24 puntos, mientras que los últimos cuatro 16. De todos modos como se ha comprobado en los cuatro años posteriores a la resolución del concurso no se ha cumplido prácticamente con ningún criterio planteado.

No existe pluralidad programática fuera de las grandes cadenas, no se ha dinamizado el sector radiofónico aragonés con proyectos de proximidad, la programación local y de cercanía es muy limitada y se han producido transferencias y cesiones de frecuencias.

Tras valorar la situación de la radio comercial de la región observamos que esta Comunidad Autónoma carece de una oferta propia de carácter aragonés, con la única excepción de la facilitada por las radios municipales y las sin licencia, ya que las desconexiones realizadas por Radio Nacional de España también son escasas.

Sin embargo, los centros dependientes de los ayuntamientos y los que carecen de autorización legal tienen una incidencia mínima en cuanto a su audiencia y sus parrillas se basan fundamentalmente en contenidos musicales, por lo que también se pone en duda que aporten una programación de proximidad diferente a la de las grandes cadenas. Asimismo, un número importante de estos dos tipos de operadores conectan durante buena parte de la jornada con alguna red estatal.

Así podemos asegurar que la oferta radiofónica de cercanía es muy reducida en Aragón y que prácticamente no se ha incrementado pese a la asignación de 32 frecuencias por parte del Gobierno regional en 1998. El hecho de que esta comunidad no cuente con una radio pública autonómica condiciona, de forma evidente, este elemento.

La idea de crear una corporación de medios públicos en Aragón, en la que pueda producirse también la participación de la empresa privada, ha vuelto a resurgir y es probable, después de los movimientos de las diversas formaciones políticas en los últimos tiempos, que el inicio de la próxima legislatura sea el momento en el que definitivamente desde el Gobierno aragonés se apueste por este hecho.

En este sentido, el pasado mes de agosto, Izquierda Unida en Aragón (IU), que durante una parte de esta legislatura ha apoyado al Gobierno formado por la 
coalición PSOE-PAR, presentó su propuesta para crear una Radio Televisión Pública Aragonesa, basada fundamentalmente en el criterio de convertirse en un servicio público más. En relación con este hecho, uno de los aspectos que IU exigió durante los meses que apoyó al Ejecutivo regional fue la creación de una radio autonómica pública, pero la posterior ruptura con el Gobierno hizo desestimar su aparición.

De esta forma, observamos que existe en Aragón un vacío en cuanto a la programación de producción propia basada en contenidos aragoneses, pese a que éste era uno de los principales aspectos que se valoraron en los criterios de selección de los adjudicatarios de las 32 emisoras comerciales que se repartieron en 1998. Además, esta situación también está condicionada por la mínima importancia de las radios municipales y la no regulación de las que ahora denominamos sin licencia y, sobre todo, por la falta de una radio pública autonómica con cobertura en todo el territorio que sí dinamizaría este medio de comunicación en la región, sobre todo, porque permitiría apostar por los contenidos de cercanía frente a los globalizados que actualmente se difunden en la mayor parte de operadores que se sintonizan en esta demarcación.

\section{Bibliografía:}

- CHAPARRO, M. (Ed.) (1997): Radio pública local y alternativa. Perspectivas. Edt. Asociación de Emisoras Municipales de Andalucía de Radio y Televisión. Jerez.

- CHICÓN, J.J. (1998): Setenta años... y un día. Historias de una radio llamada Zaragoza. Edt. Mira Editores. Zaragoza.

- DUEÑAS, J. A.; SERRANO, A. (Ed.) (1990): Historia del periodismo aragonés. Edt. Diputación de Zaragoza, Diputación de Huesca, Diputación de Teruel y Asociación de la Prensa de Zaragoza. Zaragoza.

- ESCAlANTE, G. (1986): Medio siglo de Radio Huesca. Recuerdo de una emisora. Edición a cargo del autor. Huesca.

- GUILLÉN, E. (2001): Medios y sociedad en Aragón: discursos, construcción de identidad y relaciones de poder. Tesis Doctoral. UAB. Multicopiado.

- MARTÍ, J.M. (1990): Modelos de programación radiofónica. Edt. Feed-Back. Barcelona.

- SABÉS, F. (2002): La radio y la televisión local en Aragón. Edt. Milenio. Lleida.

- SABÉS, F. (2002): Propuestas para una redefinición de la comunicación local en la convergencia mediática. Comunicación presentada al congreso Comunicación, Universidad y Sociedad del Conocimiento celebrado en Salamanca los días 24, 25 i 26 de enero. (Consultad la siguiente dirección electrónica: 
http://www.dialectus.com/colaboraciones/fernandosabesturmo/ comunicacion_local.htm)

- SABÉS, F. (2002): La radio y la televisión local en el marco del sistema audiovisual aragonés. Tesis doctoral. Edt. Servei de Publicacions de la Universitat Autónoma de Barcelona (UAB). Bellaterra. En la siguiente dirección electrónica http://www.tdcat.cesca.es/TDCat-0613102-131511/

- SABÉS, F. (2002): Clasificación de las emisoras de radio. El caso aragonés. (Consultad la siguiente dirección electrónica: http://www.dialectus.com/colaboraciones/fernandosabesturmo/clasificacion-emisoras-de-radio.htm)

- SABÉS, F. (2001): "El Sistema Radiofónico Aragonés". En Balcei, núm. 74, marzo. Edt. Ayuntamiento de Alcorisa. Alcorisa. (Teruel). Pág. 66. 